\title{
5 Series Expansions for Finite-State Markov Chains
}

Bernd Heidergott 1,3

Arie Hordijk ${ }^{2}$

Miranda van Uitert ${ }^{l}$

' Department of Economics, Vrije Universiteit Amsterdam;

2 Leiden University, Mathematical Institute;

3 Tinbergen Institute. 


\section{Tinbergen Institute}

The Tinbergen Institute is the institute for economic research of the Erasmus Universiteit Rotterdam, Universiteit van Amsterdam, and Vrije Universiteit Amsterdam.

Tinbergen Institute Amsterdam

Roetersstraat 31

1018 WB Amsterdam

The Netherlands

Tel.: $\quad+31(0) 205513500$

Fax: $\quad+31(0) 205513555$

Tinbergen Institute Rotterdam

Burg. Oudlaan 50

3062 PA Rotterdam

The Netherlands

Tel.: $\quad+31(0) 104088900$

Fax: $\quad+31(0) 104089031$

Please send questions and/or remarks of nonscientific nature to driessen@tinbergen.nl.

Most TI discussion papers can be downloaded at http://www.tinbergen.nl. 


\title{
Series Expansions for Finite-State Markov Chains
}

\author{
Bernd Heidergott $^{a}$, Arie Hordijk $\mathrm{k}^{b}$ and Miranda van Uitert $^{a}$ \\ ${ }^{[a]}$ Vrije Universiteit, Department of Econometrics \\ De Boelelaan 1105, 1081 HV Amsterdam \\ email: \{bheidergott,muitert\}@feweb.vu.nl \\ [b] Leiden University, Mathematical Institute \\ P.O.Box 9512, 2300 RA Leiden, the Netherlands \\ email: hordijk@math.leidenuniv.nl
}

\begin{abstract}
This paper provides series expansions of the stationary distribution of a finite Markov chain. This leads to an efficient numerical algorithm for computing the stationary distribution of a finite Markov chain. Numerical examples are given to illustrate the performance of the algorithm.

Key words: finite-state Markov chain - (Taylor) series expansion- measure-valued derivatives - coupled processors
\end{abstract}

\section{Introduction}

Let $P$ denote the transition kernel of a Markov chain defined on a finite statespace $\mathcal{S}$ having unique stationary distribution $\pi_{P}$. For example, think of $P$ as the embedded jump chain of an $\mathrm{M} / \mathrm{M} / 1 / \mathrm{c}$ queue, where $c$ is a finite number denoting the buffer capacity of the queue. What would be the effect on the stationary behaviour of the queue if, for example, we increased the buffer capacity or adjusted the service rate of the queue? Let $Q$ denote the Markov transition kernel of the Markov chain modeling the alternative system and assume that $Q$ has unique stationary distribution $\pi_{Q}$. The question about the effect of switching from $P$ to $Q$ on the stationary behavior is expressed by $\pi_{P}-\pi_{Q}$, the difference between the stationary distributions. Let $\|\cdot\|_{\text {tv }}$ denote the total variation norm, then the above problem can be phrased as follows: Can $\left\|\pi_{P}-\pi_{Q}\right\|_{\mathrm{tv}}$ be approximated or bounded in terms of $\|P-Q\|_{\mathrm{tv}}$ ? This is known as perturbation analysis of Markov chains (PAMC) in the literature.

In this paper we will show that $\pi_{P}-\pi_{Q}$ can be arbitrarily closely approximated by a polynomial in $(Q-P) D_{P}$, where $D_{P}$ denotes the deviation matrix associated with $P$, a precise definition will be given later on. Starting point 
is the representation

$$
\pi_{Q}=\sum_{n=0}^{k} \pi_{P}\left((Q-P) D_{P}\right)^{n}+\pi_{Q}\left((Q-P) D_{P}\right)^{k+1},
$$

for any $k \geq 0$, a proof of which will be given in Section 3.1. This series expansion of $\pi_{Q}$ provides the means of approximating $\pi_{Q}$ by $Q$ and entities given via the $P$ Markov chain only. A straightforward approach of achieving this would be to apply the total variation norm to (1) and to conclude that

$$
\left\|\pi_{Q}-\sum_{n=0}^{k} \pi_{P}\left((Q-P) D_{P}\right)^{n}\right\|_{\mathrm{tv}} \leq\left\|(Q-P) D_{P}\right\|_{\mathrm{tv}}^{k+1},
$$

for any $k$. However, this is to no avail since the total variation norm of $(Q-P) D_{P}$ is typically larger than one. In this paper, we propose to work with the weighted supremum norm, denoted by $\|\cdot\|_{v}$, where $v$ is some vector with positive non-zero elements, and for any $w \in \mathbb{R}^{\mathcal{S}}$

$$
\|w\|_{v} \stackrel{\text { def }}{=} \sup _{i \in \mathcal{S}} \frac{|w(i)|}{v(i)} .
$$

We will show that

$$
\pi_{Q}(s)-\left(\sum_{n=0}^{k} \pi_{P}\left((Q-P) D_{P}\right)^{n}\right)(s) \leq d\left\|(Q-P) D_{P}\right\|_{v}^{k+1},
$$

for any $k \in \mathbb{N}$ and any $s \in \mathcal{S}$, where $v$ can be any vector satisfying $v(s) \geq 1$ for $s \in \mathcal{S}$, and $d$ is some finite computable constant. In particular, the above error bound can be computed without knowledge of $\pi_{Q}$. The key idea of our approach is solve the optimization problem

subject to

$$
\min \left\|\left((Q-P) D_{P}\right)^{k}\right\|_{v}
$$

$$
v(s) \geq 1 \text { for } s \in \mathcal{S} .
$$

The solution $v^{*}$ of the above optimization problem can be interpreted as the optimal measure of the rate of convergence of the series in (1). Moreover, the series in (1) tends to converge extremely fast which is due to the fact that in many examples $v^{*}$ be found such that $\left\|(Q-P) D_{P}\right\|_{v^{*}} \ll 1$. Best to our knowledge, the limit of the series (1) first appeared in [2], however, neither upper bounds for the remainder term nor numerical examples were given there. 
The use of series expansion for computational purposes is not new. It has been used in various fields for different purposes, for instance in the field of linear algebra [3]. In [1] the authors derive the power series for the stationary distribution in a slightly different way than [7] and the approach we take in this paper, but they don't use it for real problems or real computations. The novelty of our work is the fact that we combine the ideas obtained, and extend them such that problems can be really solved.

The series expansion for the finite-state Markov chains in this paper is derived in a very elegant manner using the Poisson equation. We remark that this series expansion holds in a very general format under proper conditions. The derivation of this has been done in [7], which is a generalization of [2].

The work presented in this paper is part of a major research project on numerical algorithms based on series expansions of Markov chains. The present paper establishes the main theoretical results. In a followup paper, we will study our methodology for large scaled problems.

The paper is organized as follows. Section 2 presents basic facts on finite Markov chains. The series expansion (1) is discussed in Section 3. In particular, numerical examples are provided. Section 4 presents the extension to convex combinations of Markov chains.

\section{Preliminaries on finite Markov chains}

Let $\mathcal{S}$ denote a finite set with $0<S<\infty$ elements. For notational convenience we will identify $\mathcal{S}$ with the set $\{1, \ldots, S\}$. We consider Markov kernels on state space $\mathcal{S}$. Such a Markov kernel, say $P$, can be written as square matrix $P \in[0,1]^{S \times S}$. Element $(i, j)$ of $P$ is denoted by $P(i, j)$ and represents the probability of jump from state $i$ to state $j$, which implies $\sum_{j \in \mathcal{S}} P(i, j)=1$, for all $i \in \mathcal{S}$. The probability to go from state $i$ to state $j$ in $n$ steps is denoted by $P^{n}(i, j)$, where the Markov kernel $P^{n}$ is simply obtained from taking the $n$th power of $P$. Provided it exists, we denote the unique stationary distribution of $P$ by $\pi_{P}$ and its ergodic projector by $\Pi_{P}$, that is, for any distribution $\mu$ it holds that $\mu \Pi_{P}=\pi_{P}$. In order to simplify the notation, we will -with slight abuse of notation- identify $\pi_{P}$ and $\pi_{Q}$ with $\Pi_{P}$ and $\Pi_{Q}$, respectively.

Throughout the paper we assume that $P$ is aperiodic and unichain, which means that there is one closed irreducible set of states, and a (possibly empty) set of transient states. We write $|A|(i, j)$ to denote the $(i, j)$ th element of the matrix of absolute values of $A \in \mathbb{R}^{S \times S}$, and additionally we use the notation 
$|A|$ for the matrix of absolute values of $A$.

The main tool for our analysis is the weighted supremum norm, also called $v$-norm, as defined in (2). For a matrix $A \in \mathbb{R}^{S \times S}$ the $v$-norm is given by

$$
\|A\|_{v} \stackrel{\text { def }}{=} \sup _{i,\|w\|_{v} \leq 1} \frac{\sum_{j=1}^{S}|A(i, j) w(j)|}{v(i)} .
$$

Observe that

$$
\|A\|_{v}=\sup _{i} \frac{\sum_{j=1}^{S}|A|(i, j) v(j)}{v(i)},
$$

which is due to $\|v\|_{v}=1$ and the fact that $\|w\|_{v} \leq 1$ implies $|w(i)| \leq v(i)$, for $i \in \mathcal{S}$. Obviously, this implies

$$
v(i)\|A\|_{v} \geq \sum_{j=1}^{S}|A|(i, j) v(j), \quad i \in \mathcal{S},
$$

and hence, using $v(i) \geq 1, i \in \mathcal{S}$, we obtain

$$
\max _{j \in \mathcal{S}}|A|(i, j) \leq\|A\|_{v} v(i), \quad i \in \mathcal{S} .
$$

From (4) it readily follows that $v$-norm convergence to 0 implies elementwise convergence to 0 . More precisely, let $\left\{A(n) \in \mathbb{R}^{S \times S}, n=1,2, \ldots\right\}$ be given such that $\lim _{n \rightarrow \infty}\|A(n)\|_{v}=0$ exists, then $\lim _{n \rightarrow \infty}|A(n)|(i, j)=0$ exists $\forall i, j \in \mathcal{S}$. Next we introduce $v$-geometric ergodicity (also called $v$-normed ergodicity) of $P_{\theta}$ at $\theta \in[0,1]$.

Definition 2.1 A Markov chain $P$ is v-geometric ergodic if $c<\infty, \beta<1$ and $N<\infty$ exist such that

$$
\left\|P^{n}-\Pi_{P}\right\|_{v} \leq c \beta^{n}
$$

for all $n \geq N$.

The following lemma shows that any finite-state aperiodic Markov chain is $v$-geometric ergodic.

Lemma 2.2 For finite-state and aperiodic $P$ a finite number $N$ exists such that

$$
\left\|P^{n}-\Pi_{P}\right\|_{v} \leq c \beta^{n}
$$

for all $n \geq N$, where $c<\infty$ and $\beta<1$. 
Proof Because of the finite state space and aperiodicity,

$$
\lim _{n \rightarrow \infty} P^{n}(i, j)=\Pi_{P}(i, j), \quad i, j \in \mathcal{S} .
$$

Moreover, it is possible to take $N$ sufficiently large such that

$$
\forall i \in \mathcal{S}: \quad \frac{\sum_{j=1}^{S}\left|P^{N}-\Pi_{P}\right|(i, j) v(j)}{v(i)}<\epsilon,
$$

with $\epsilon<1$. Because $P^{n}-\Pi_{P}=\left(P-\Pi_{P}\right)^{n}$ for $n \geq 1$, this means that

$$
\left\|\left(P-\Pi_{P}\right)^{N}\right\|_{v}<\epsilon .
$$

Taking appropriate integers $n, k$ and $l$, such that $n=k N+l$ we then find

$$
\left\|P^{n}-\Pi_{P}\right\| \leq \epsilon^{k}\left\|P^{l}-\Pi_{P}\right\|_{v},
$$

using norm inequalities. Define

$$
c \stackrel{\text { def }}{=} \sup _{l=0, \ldots, N-1}\left\|P^{l}-\Pi_{P}\right\|_{v},
$$

and $\beta \stackrel{\text { def }}{=} \epsilon^{\frac{1}{N+1}}$. Since $\epsilon<1$, it follows that $\beta<1$, which implies

$$
\epsilon^{k}=\beta^{k N+k} \leq \beta^{k N+l}=\beta^{n},
$$

where we use the fact that $0 \leq l<k$. Inserting (7) and (8) in (6), we obtain the stated.

We write $D_{P}$ for the deviation matrix associated with $P$; in symbols:

$$
D_{P}=\sum_{m=0}^{\infty}\left(P^{m}-\Pi_{P}\right) .
$$

Note that $D_{P}$ is finite for any aperiodic finite-state Markov chain, see Lemma 2.2. Moreover, the deviation can be rewritten as

$$
D_{P}=\sum_{m=0}^{\infty}\left(P-\Pi_{P}\right)^{m}-\Pi_{P},
$$

where $\sum_{m=0}^{\infty}\left(P-\Pi_{P}\right)^{m}$ is often referred to as the group inverse, see for instance $[2,4]$. A general definition which is valid for any, possibly periodic Markov chain, can be found in, e.g., [12]. 


\section{Series Expansions}

We are interested in the performance of a system when some of its parameters or characteristics are changed. The system as given is modeled as a Markov chain with kernel $P$, the changed system with kernel $Q$. We assume that both Markov chains have a common finite state space $\mathcal{S}$ as defined earlier, i.e., $P, Q \in[0,1]^{S \times S}$. Note that sometimes states have to be added to $Q$ or $P$ in order to achieve this. We also assume, as indicated earlier, that both Markov kernels are aperiodic and unichain. The goal of Section 3.1 is to obtain the stationary distribution of $Q$, denoted by $\pi_{Q}$, via a series expansion in $P$. In Section 3.2 we comment on the speed of convergence of this series. When applying these concepts to actually compute the stationary distribution, we have to solve the optimization problem stated in (3). We explain in Section 3.3 how to find an optimal solution. We summarize our results in an algorithm, presented in Section 3.4. Finally, we illustrate our approach with numerical examples in Section 3.5.

\subsection{Series representation for $\pi_{Q}$}

Let $P$ be unichain (not necessarily finite). Elementary calculation shows (use the definition of $D_{P}$ in $\left.(9)\right)$ :

$$
(I-P) D_{P}=I-\Pi_{P} .
$$

This is the Poisson equation in matrix format. Multiply this equation by $\Pi_{Q}$. Since $P$ is unichain it holds $\Pi_{Q} \Pi_{P}=\Pi_{P}$, and we obtain

$$
\Pi_{Q}(I-P) D_{P}=\Pi_{Q}-\Pi_{P} .
$$

Using that $\Pi_{Q}=\Pi_{Q} Q$, we obtain

$$
\Pi_{Q}=\Pi_{P}+\Pi_{Q}(Q-P) D_{P} .
$$

Inserting (10) for $\Pi_{Q}$ in the right-hand side of (10) we obtain

$$
\Pi_{Q}=\Pi_{P}+\Pi_{P}(Q-P) D_{P}+\Pi_{Q}\left((Q-P) D_{P}\right)^{2} .
$$

Repeating this step $k$ times yields,

$$
\Pi_{Q}=\Pi_{P} \sum_{n=0}^{k}\left((Q-P) D_{P}\right)^{n}+\Pi_{Q}\left((Q-P) D_{P}\right)^{k+1},
$$


for $k \geq 0$. Based on the above equation, we introduce the following notation. Let $k \geq 0$, then $H(k)$, with

$$
H(k) \stackrel{\text { def }}{=} \Pi_{P} \sum_{n=0}^{k}\left((Q-P) D_{P}\right)^{n},
$$

is called a series approximation of degree $k$ for $\Pi_{Q}, T(k)$, with

$$
T(k) \stackrel{\text { def }}{=} \Pi_{P}\left((Q-P) D_{P}\right)^{k},
$$

denotes the $k$ th element of $H(k)$, and $R(k)$, with

$$
R(k) \stackrel{\text { def }}{=} \Pi_{Q}\left((Q-P) D_{P}\right)^{k+1},
$$

is called the remainder term. Notice that the remainder term $R(k)$ almost equals the $(k+1)$ st term of $H(k+1)$, i.e., $T(k+1)$, except for the prefactor, which is $\Pi_{Q}$ in $R(k)$ and $\Pi_{P}$ in $T(k+1)$. The quality of the approximation provided by $H(k)$ is given through the remainder term $R(k)$. This issue is discussed in the next section.

\subsection{Convergence of the series}

In this section we investigate the limiting behavior of $H(k)$ as $k$ tends to $\infty$. We first introduce our main technical condition.

(C) There exists a finite number $N$ such that we can find $\delta_{N} \in(0,1)$ which satisfies:

$$
\left\|\left((Q-P) D_{P}\right)^{N}\right\|_{v}<\delta_{N}
$$

and we set

$$
c_{\delta_{N}} \stackrel{\text { def }}{=} \frac{1}{1-\delta_{N}}\left\|\sum_{k=0}^{N-1}\left((Q-P) D_{p}\right)^{k}\right\|_{v} .
$$

Lemma 3.1 The following assertions are equivalent:

(i) The series $\sum_{k=0}^{\infty}\left((Q-P) D_{P}\right)^{k}$ is convergent.

(ii) (C)

(iii) There are $\kappa$ and $\delta<1$ such that $\left\|\left((Q-P) D_{P}\right)^{k}\right\|_{v} \leq \kappa \delta^{k}$ for any $k$.

(iv) There are $N$ and $\delta \in(0,1)$ such that $\left\|\left((Q-P) D_{P}\right)^{k}\right\|_{v}<\delta^{k}$ for any $k \geq N$. 
Proof We first show that (i) implies (ii). Provided that $\sum_{k=0}^{\infty}\left((Q-P) D_{P}\right)^{k}$ converges, it holds that

$$
\lim _{k \rightarrow \infty}\left[\left((Q-P) D_{P}\right)^{k}\right](i, j)=0
$$

for $i, j \in \mathcal{S}$. Since $\mathcal{S}$ is finite, it is possible to take $k$ sufficiently large such that

$$
\frac{\sum_{j \in \mathcal{S}}\left|\left((Q-P) D_{P}\right)^{k}\right|(i, j) v(j)}{v(i)}<1,
$$

which implies (C).

Now suppose that $(\mathbf{C})$ holds true, and set $\varepsilon \stackrel{\text { def }}{=}\left\|\left((Q-P) D_{P}\right)^{N}\right\|_{v}$. Let $k, m, l$ be integers such that $k=m N+l$. Then,

$$
\begin{aligned}
\left\|\left((Q-P) D_{P}\right)^{k}\right\|_{v} & =\left\|\left((Q-P) D_{P}\right)^{m N+l}\right\|_{v} \\
& \leq\left\|\left((Q-P) D_{P}\right)^{m N}\right\|_{v}\left\|\left((Q-P) D_{P}\right)^{l}\right\|_{v} \\
& \leq \varepsilon^{m}\left\|\left((Q-P) D_{P}\right)^{l}\right\|_{v} .
\end{aligned}
$$

Set

$$
\kappa \stackrel{\text { def }}{=} \sup _{i=0, \ldots, N-1}\left\|\left((Q-P) D_{P}\right)^{i}\right\|_{v}
$$

and $\delta=\varepsilon^{\frac{1}{N+1}}$. By (ii), $\varepsilon<1$ and thus $\delta<1$. Hence, the following holds

$$
\delta^{k}=\delta^{m N+l} \geq \delta^{m N+N}=\varepsilon^{m},
$$

where we use the fact that $0 \leq l<N$. Inserting the above in (13) yields (iii).

Suppose (iii) holds. In the case that $\kappa \leq 1$, (iv) is immediate. In the case $\kappa>1$, let $N$ and $\beta<1$ be such that

$$
\kappa^{1 / n} \delta \leq \beta, \quad n \geq N .
$$

Then,

$$
\kappa \delta^{n} \leq \beta^{n}, \quad n \geq N
$$

and (iv) is satisfied.

Suppose (iv), then

$$
\sum_{k=0}^{\infty}\left\|\left((Q-P) D_{P}\right)^{k}\right\|_{v} \leq \frac{\delta^{N}}{1-\delta}+\sum_{k=0}^{N-1}\left\|\left((Q-P) D_{P}\right)^{k}\right\|_{v} .
$$

Hence, the series is convergent with respect to the $v$-norm. Since $v$-norm convergence implies elementwise convergence, (i) follows. 
Remark 3.2 Note that the fact that the maximal eigenvalue of $\left|(Q-P) D_{P}\right|$ is smaller than 1 is not necessary for $\sum_{k=0}^{\infty}\left((Q-P) D_{P}\right)^{k}$ to converge, which stems from the fact that $\left((Q-P) D_{P}\right)^{k}$ has positive and negative entries.

The following lemma establishes an upper bound for the remainder term that is independent of $\Pi_{Q}$.

Lemma 3.3 Under (C) it holds that

(i) $\|R(k-1)\|_{v} \leq c_{\delta_{N}}\|T(k)\|_{v}$ for all $k$,

(ii) $\lim _{k \rightarrow \infty} H(k)=\Pi_{Q}$.

Proof By definition we have

$$
\|R(k-1)\|_{v}=\left\|\pi_{Q}-\sum_{l=0}^{k-1} T(l)\right\|_{v}=\left\|\sum_{l=k}^{\infty} T(l)\right\|_{v} .
$$

This is obviously equal to

$$
\begin{aligned}
\left\|\Pi_{P} \sum_{l=k}^{\infty}\left((Q-P) D_{P}\right)^{l}\right\|_{v} & =\left\|\Pi_{P}\left((Q-P) D_{P}\right)^{k} \sum_{l=0}^{\infty}\left((Q-P) D_{P}\right)^{l}\right\|_{v} \\
& \leq\|T(k)\|_{v}\left\|\sum_{l=0}^{\infty}\left((Q-P) D_{P}\right)^{l}\right\|_{v} \\
& \leq\|T(k)\|_{v}\left\|\sum_{l=0}^{\infty}\left((Q-P) D_{P}\right)^{l N}\right\|\left\|_{v}\right\| \sum_{k=0}^{N-1}\left((Q-P) D_{P}\right)^{k} \|_{v} \\
& \leq \frac{1}{1-\delta_{N}}\|T(k)\|\left\|\sum_{k=0}^{N-1}\left((Q-P) D_{P}\right)^{k}\right\|_{v},
\end{aligned}
$$

which proves the first part of the lemma.

With (4) it is sufficient to show that we have $v$-norm convergence for $\Pi_{Q}-H(k)$. Using claim (i) we obtain

$$
0 \leq\|R(k)\|_{v} \leq c_{\delta_{N}}\left\|\Pi_{P}\right\|_{v}\left\|\left((Q-P) D_{P}\right)^{k+1}\right\|_{v} .
$$

By Lemma 3.1 (iv) the right hand-side in the above inequality converges to zero. This proves the claim.

An example where the series $H(k)$ fails to converge is illustrated in the following. 
Example 3.4 Suppose the state space $\mathcal{S}=\{0,1, \ldots, S\}$. Let $P(i, j)=Q(i, j)$ for $i \neq 0$ and all $j, P(0,0)=Q(0, S)=1$, and $P(i, i-1)=1$ for $i \neq 0$. Then

$$
\begin{aligned}
& \Pi_{P}(i, 0)=1 \\
& D_{P}(i, j)=\sum_{k=0}^{\infty}\left(P^{k}-\Pi_{P}\right)(i, j)=1,
\end{aligned}
$$

for $i \geq j \geq 1$ and $=0$ for $i \geq 1, i<j$

$$
\begin{aligned}
& D_{P}(i, 0)=-(i-1) \\
& D_{P}(0, j)=0
\end{aligned}
$$

for all $j \geq 0$. Hence, $((Q-P) D)(i, j)=0$ for $i \neq 0$ and

$$
((Q-P) D)(0, j)=-(S-j)
$$

for $j \geq 1$ and

$$
((Q-P) D)(0,0)=-(S-1)
$$

and we have that

$$
((Q-P) D)^{k}(0,0)=(-(S-1))^{k} .
$$

Therefore, the series is divergent.

Remark 3.5 Provided that $\operatorname{det}\left(\mathrm{I}-(\mathrm{Q}-\mathrm{P}) \mathrm{D}_{\mathrm{P}}\right) \neq 0$, (10) determines $\pi_{Q}$ uniquely and one can obtain $\pi_{Q}$ from

$$
\pi_{Q}=\Pi_{P}\left(I-(Q-P) D_{P}\right)^{-1} .
$$

Moreover, provided that the limit

$$
\lim _{k \rightarrow \infty} H(k)=\lim _{k \rightarrow \infty} \pi_{P} \sum_{n=0}^{\infty}\left((Q-P) D_{P}\right)^{n}
$$

exists (see Lemma 3.1 for sufficient conditions), it yields $\pi_{Q}$ as $\pi_{P} \sum_{n=0}^{\infty}((Q-$ P) $\left.D_{P}\right)^{n}$; see Lemma 3.3.

Remark 3.6 Note that a sufficient (but not necessary) condition for (C) is

$$
\left\|(Q-P) D_{P}\right\|_{v}<\delta, \quad \delta<1 .
$$


We will present examples for which it turns out that $\left\|(Q-P) D_{P}\right\|_{v}>1$, although $(\mathbf{C})$ is satisfied for $N \geq 2$. In [1,3] it is even assumed that

$$
\|(Q-P)\|_{v}<g_{1}
$$

with $g_{1}>0$ a finite constant, and

$$
\left\|D_{P}\right\|_{v}<\frac{c}{1-\beta}
$$

with $c>0$ and $0<\beta<1$ finite constants. If

$$
\frac{g_{1} c}{1-\beta}<1
$$

then (14) and hence (C) is clearly fulfilled. However, we will illustrate with some examples presented in Sections 3.5 and 4.1 that we cannot find $v$ such that both (15), (16), and (17) hold. Hence, for numerical purposes these conditions are too strong.

\subsection{Bounding the remainder term}

Until now we haven't specified $v$. The quality of approximation by $H(k-1)$ is given by the remainder term $R(k-1)$ and in applications $v$ should be chosen such that it minimizes $c_{\delta_{N}}\|T(k)\|_{v}$, thus minimizing our upper bound for the remainder term. Since $c_{\delta_{N}}$ is a constant, we focus on $T(k)$ for finding an optimal upper bound. Specifically, we have to find a bounding vector $v$ that minimizes $\|T(k)\|_{v}$ uniformly w.r.t. $k$. As the following theorem shows, the unit vector, denoted by $\mathbf{1}$, with all components equal to one, yields the minimal value for $\|T(k)\|_{v}$ for any $k$. The proof of the theorem is given in the Appendix.

Theorem 3.7 The unit vector 1 minimizes $\|T(k)\|_{v}$ uniformly over $k$, i.e.,

$$
\forall k \geq 1: \quad \inf _{v}\|T(k)\|_{v}=\|T(k)\|_{1} .
$$

Remark 3.8 As for the results in [1, 3], following the line of argument put forward in the Appendix, it can be shown that the smallest $g_{1}$ in (15) is the maximal eigenvalue of $|(Q-P)|$, and the smallest $\frac{c}{1-\beta}$ is precisely the maximal eigenvalue of $\left|D_{P}\right|$. Again we note that often the product of these maximal eigenvalues is not smaller than 1. In Sections 3.5 and 4.1 we will present examples for which $\frac{c g_{1}}{1-\beta}>1$. If this is the case, then according to [1, 3] we cannot decide whether the series $H(k)$ converges to $\Pi_{Q}$. Hence, their condition is too restrictive for numerical purposes. 


\subsection{Algorithm}

In this section we describe a numerical approach to computing our upper bound for the remainder term $R(k)$. We search for $N$ such that $1>\delta_{N} \stackrel{\text { def }}{=}\left\|\left((Q-P) D_{P}\right)^{N}\right\|_{\mathbf{1}}$, which implies that $(\mathbf{C})$ holds for $N$ and $\delta_{N}$. Then the upper bound for $R(k)$ is obtained from $c_{\delta_{N}}\left\|\left((Q-P) D_{P}\right)^{k+1}\right\|_{1}$.

Based on the above, we can now describe an algorithm that yields an approximation for $\pi_{Q}$ with $\epsilon$ precision. The algorithm has two main parts. First $c_{\delta_{N}}$ is computed. Then, the series can be computed in an iterative way until a predefined level of precision is reached.

\section{Algorithm 1}

Chose precision $\epsilon>0$. Set $k=1, T(1)=\Pi_{P}(Q-P) D_{P}$ and $H(0)=\Pi_{P}$.

Step 1: Find $N$ such that $\left\|\left((Q-P) D_{P}\right)^{N}\right\|_{1}<1$. Set $\delta_{N}=\|((Q-$ P) $\left.D_{P}\right)^{N} \|_{1}$ and compute

$$
c_{\delta_{N}}=\frac{1}{1-\delta_{N}}\left\|\sum_{k=0}^{N-1}\left((Q-P) D_{p}\right)^{k}\right\|_{1} .
$$

Step 2: If

$$
c_{\delta}\|T(k)\|_{1}<\epsilon
$$

the algorithm terminates and $H(k-1)$ yields the desired approximation. Otherwise, go to step 3.

Step 3: Set $H(k)=H(k-1)+T(k)$. Set $k:=k+1$ and $T(k)=T(k-1)(Q-P) D_{P}$. Go to step 2 .

Lemma 3.9 Provided that $\sum_{k-0}^{\infty}\left((Q-P) D_{P}\right)^{k}$ is finite, Algorithm 1 terminates in a finite number of steps.

Proof By Lemma 3.1, finiteness of $\sum_{k-0}^{\infty}\left((Q-P) D_{P}\right)^{k}$ implies (C). Thus, we know from Lemma 3.3 that $\|R(k)\|_{\mathbf{1}} \leq c_{\delta_{N}}\|T(k+1)\| \mathbf{1}$ for any $k$. Since

$$
\|T(k+1)\|_{\mathbf{v}} \leq\left\|\Pi_{P}\right\|_{\mathbf{1}}\left\|\left((Q-P) D_{P}\right)^{k}\right\|_{\mathbf{1}},
$$


the proof of the claim follows from the fact that $\lim _{k \rightarrow \infty}\left\|\left((Q-P) D_{P}\right)^{k}\right\|_{1}=0$, see Lemma 3.1.

\subsection{Numerical Examples}

Consider a model where customers have to be served first at station 1 and after having successfully completed service, move on to station 2 to receive service there. We assume that customers receive a successful service at station 1 with probability $1-p$, i.e., a fraction $p$ of the customers has to go through station 1 again. Customers arrive at the system according to a Poisson process with rate $\lambda$, and are served at station $i, i=1,2$ with an exponential service time with rate $\mu_{i}$. We assume the waiting capacity at both stations to be finite, i.e., $b_{i}$ customers can be at station $i$ (including the customer in service), and that customers that find upon arrival or service completion no free buffer places at the next station are lost. The current system with parameters $\lambda, \mu_{1}, \mu_{2}, b_{1}$ and $b_{2}$ is modeled as a uniformized embedded Markov chain with kernel $P$, and its stationary distribution $\pi_{P}$ is known. We take in all examples $p=0.25, \mu_{1}=2, \mu_{2}=2$, and $b_{1}=10=b_{2}$. We are interested in the probability that customers find station 2 full; we call this probability by overflow probability and denote it by $p_{\lambda}$.

We want to investigate what happens when the waiting capacity of station 2 is expanded to $b_{2}+1$. Hence, kernel $Q$ represents the uniformized embedded Markov chain of the system with an additional buffer place at station 2. Note that we created a phantom state $b_{2}+1$ in the $P$-kernel, from which a jump to $b_{2}$ occurs with probability 1 . In all numerical examples $N$ turned out to be 1 . We denote the prediction for $p_{\lambda}$ based on $H(k)$ by $p_{\lambda}(k)$. Figure 1 shows the absolute relative error (in formula, $\left|p_{\lambda}-p_{\lambda}(k)\right| / p_{\lambda}$ ) for different values of $k$ and different values for $\lambda$, i.e., different traffic loads. As can be seen, the overflow probability can be predicted within an error of 1 percent through $H(5)$, and in the light-traffic case even $H(4)$ is sufficient.

We now turn to the numerical behavior of our upper bound for the remainder term. For given $\lambda$, denote by $r_{\lambda}(k)$ the upper bound for $R(k)$ given in Lemma 3.3. Figure 2 illustrates the relative error of our upper bound (in formula, $\left.\left(r_{\lambda}(k)-\left(p_{\lambda}-p_{\lambda}(k)\right)\right) / p_{\lambda}\right)$ or various values of $\lambda$ and different values of $k$. For $\lambda=0.25$, we obtained (C) for $N=3$ and $c_{\delta_{N}}=11.17$; for $\lambda=1.0$, we obtained (C) for $N=4$ and $c_{\delta_{N}}=4.65$; and for $\lambda=1.75$, we obtained (C) for $N=5$ and $c_{\delta_{N}}=15.397$. 


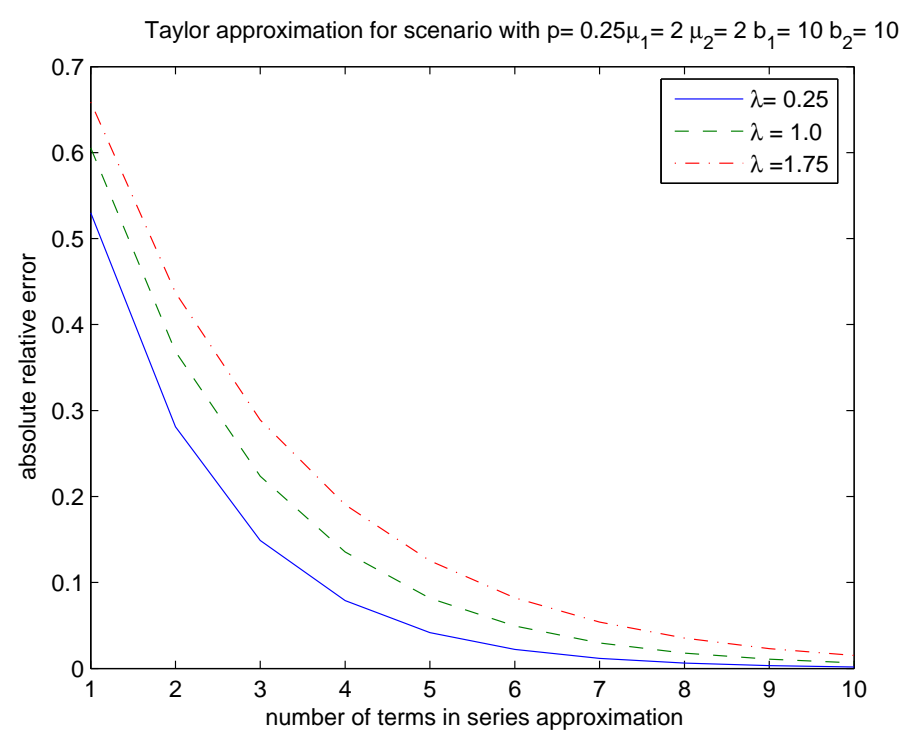

Figure 1: Absolute relative error

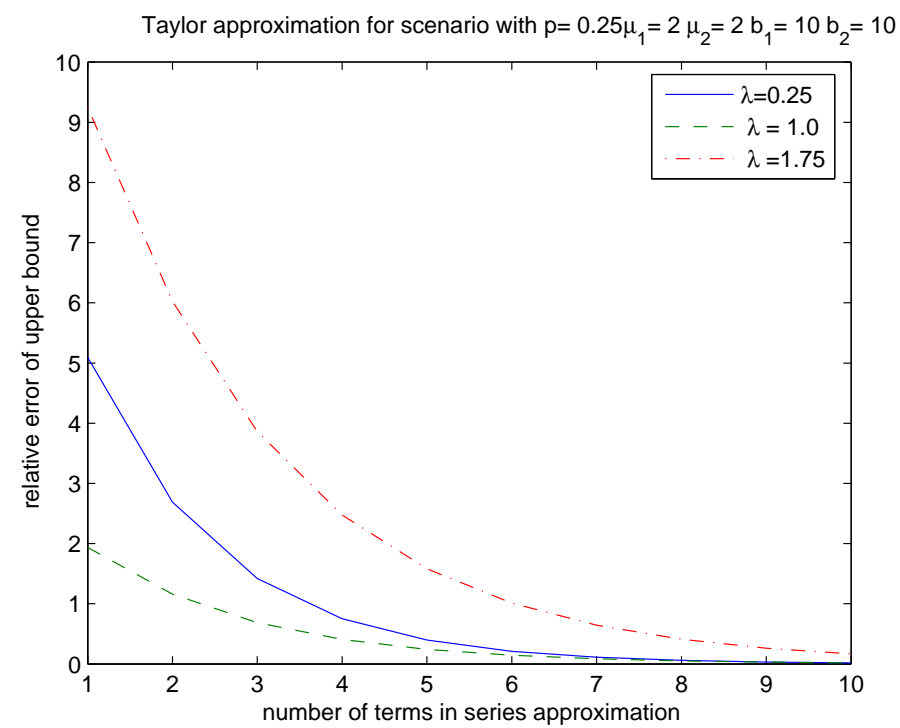

Figure 2: Relative error of the bound of the remainder term 


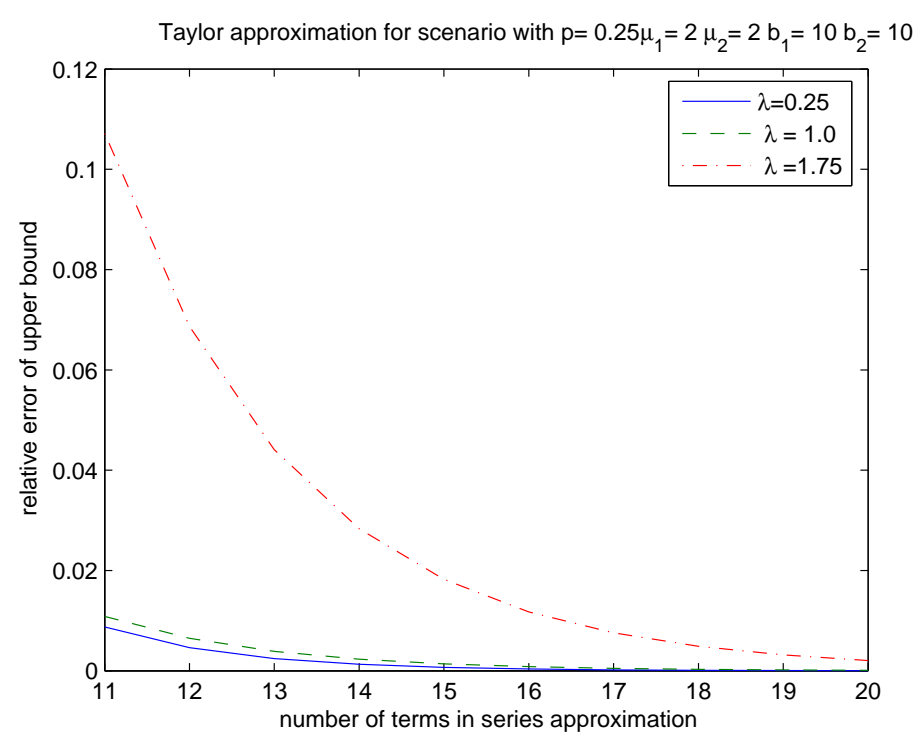

Figure 3: Relative error of the bound of the remainder term

To illustrate the decay of the relative error induced by the upper bound for the remainder term, we show in Figure 3 relative error of our upper bound for values of $k$ between 11 and 20 .

Remark 3.10 Let us turn to the condition of [1, 3]. For $p=0.5$ for instance the smallest $g_{1}$ is 0.6718 and the smallest $\frac{c}{1-\beta}$ is 6.5557 , and hence, $\frac{c g_{1}}{1-\beta}>1$, although (C) with $N=1$.

\section{A Power Series Approach}

Let $P$ and $Q$ be given as in Section 2. In this section study the convex combination

$$
P_{\theta}=(1-\theta) P+\theta Q, \quad \theta \in[0,1],
$$

of the two chains. We are now interested in the effect of changing $\theta$ to $\theta+\Delta$ on the stationary distribution of $P_{\theta}$, denoted by $\pi_{\theta}$, and the corresponding projective operator, denoted by $\Pi_{\theta}$, respectively. Following the same procedure as in Section 3.1, we obtain

$$
\Pi_{\theta+\Delta}=\Pi_{\theta} \sum_{n=0}^{k}\left(\left(P_{\theta+\Delta}-P_{\theta}\right) D_{\theta}\right)^{n}+\Pi_{\theta+\Delta}\left(\left(P_{\theta+\Delta}-P_{\theta}\right) D_{\theta}\right)^{k+1},
$$

for $k \geq 0$. From (19) it is easily seen that

$$
P_{\theta+\Delta}-P_{\theta}=\Delta(Q-P)
$$


so that the above series can be written as

$$
\Pi_{\theta+\Delta}=\Pi_{\theta} \sum_{n=0}^{k} \Delta^{n}\left((Q-P) D_{\theta}\right)^{n}+\Pi_{\theta+\Delta} \Delta^{k+1}\left((Q-P) D_{\theta}\right)^{k+1},
$$

for $k \geq 0$. For $k \geq 0$ we define the series approximation $H_{\theta, \Delta}(k)$ by

$$
H_{\theta, \Delta}(k) \stackrel{\text { def }}{=} \Pi_{\theta} \sum_{n=0}^{k} \Delta^{n}\left((Q-P) D_{P_{\theta}}\right)^{n}
$$

where we denote the $k$ th element of the above series is denoted by

$$
T_{\theta, \Delta}(k) \stackrel{\text { def }}{=} \Delta^{k} \Pi_{\theta}\left((Q-P) D_{P_{\theta}}\right)^{k},
$$

and remainder term $R_{\Delta}(k)$ is given through

$$
R_{\theta, \Delta}(k) \stackrel{\text { def }}{=} \prod_{\theta+\Delta} \Delta^{k+1}\left((Q-P) D_{P_{\theta}}\right)^{k+1} .
$$

For the series $H_{\theta, \Delta}(k)$ to converge, $\Delta^{k} T_{\theta, \Delta}(k)$ has to converge to zero as $k$ tends to $\infty$. This leads to the following adaptation of $(\mathbf{C})$.

(C) $(\theta)$ There exist finite numbers $N$ and $\delta_{N}^{\theta} \in(0,1)$ such that

$$
\left\|\left((Q-P) D_{\theta}\right)^{N}\right\|_{v} \leq \delta_{N}^{\theta},
$$

and we set

$$
c_{\delta_{N}^{\theta}} \stackrel{\text { def }}{=} \frac{1}{1-\delta_{N}^{\theta}}\left\|\sum_{k=0}^{N-1}\left((Q-P) D_{\theta}\right)^{k}\right\|_{v} .
$$

Condition (C) $(\theta)$ implies that $H_{\theta, \Delta}(k)$ converges at least for all $\Delta$ with $|\Delta|<$ $1 / \delta_{N}^{\theta}$. The following lemma is a straightforward adaptation of the result for series expansion.

Lemma 4.1 For $\theta \in[0,1]$, let $(\mathbf{C})(\theta)$ be satisfied for some $\delta_{N}^{\theta}$. Then it holds for all $|\Delta|<1 / \delta_{N}^{\theta}$ that

(i) $\left\|R_{\theta, \Delta}(k-1) \leq c_{\delta_{N}^{\theta}}\right\| T_{\theta, \Delta}(k) \|_{v}$ for all $k$;

(ii) $\lim _{k \rightarrow \infty} H_{\theta, \Delta}(k)=\Pi_{\theta+\Delta}$.

The above leads to the following algorithm that yields an approximation for $\pi_{\theta+\Delta}$ with $\epsilon$ precision, where we make use of Theorem 3.7. 


\section{Algorithm 2}

Chose precision $\epsilon>0$. Set $k=1, T_{\theta, \Delta}(1)=\Delta \Pi_{\theta}(Q-P) D_{\theta}$ and $H_{\theta, \Delta}(0)=\Pi_{\theta}$.

Step 1: Find $N$ such that $\left\|\left((Q-P) D_{\theta}\right)^{N}\right\|_{1}<1$. Set $\delta_{N}^{\theta}=\left\|\left((Q-P) D_{\theta}\right)^{N}\right\|_{1}$ and compute

$$
c_{\delta_{N}^{\theta}}=\frac{1}{1-\delta_{N}^{\theta}}\left\|\sum_{k=0}^{N-1}\left((Q-P) D_{\theta}\right)^{k}\right\|_{1} .
$$

Step 2: If

$$
c_{\delta_{N}^{\theta}}\left\|T_{\theta, \Delta}(k)\right\|_{1}<\epsilon,
$$

the algorithm terminates and $H_{\theta, \Delta}(k-1)$ yields the desired approximation. Otherwise, go to step 3.

Step 3: Set

$$
T_{\theta, \Delta}(k+1)=\Delta T_{\theta, \Delta}(k)(Q-P) D_{P_{\theta}}
$$

and $H_{\theta, \Delta}(k)=H_{\theta, \Delta}(k-1)+T_{\theta, \Delta}(k)$. Let $k:=k+1$ and go to step 2.

The above algorithm is not guaranteed to yield the desired approximation, which stems from the fact that $|\Delta|$ may lay outside the radius of convergence of the series in (21). However, $1 / \delta_{N}^{\theta}$ as computed by the above algorithm yields a lower bound for radius of convergence of $H_{\theta, \Delta}(k)$ and thus an indication of the maximal value of $|\Delta|$.

Remark 4.2 Taking $\theta=0$ and $\Delta=1$ we are in the situation of Section 3.1. In symbols, $H(k)=H_{\theta, 1}(k)$, for $k \geq 0$.

The series given in (20) is obviously a power series. Moreover, it is shown in [7] that under v-geometric ergodicity of $P_{\theta}$, the term $\Pi_{\theta}\left((Q-P) D_{P_{\theta}}\right)^{n}$ is equal to $\frac{\mathrm{d} \Pi_{\theta}}{\mathrm{d} \theta^{n}}$. This means that (20) is a Taylor series.

Hence, Lemma 4.1 yields a lower bound for the radius of convergence of the Taylor series $H_{\theta, \Delta}$, i.e., $H_{\theta, \Delta}$ converges to the right function on $\Delta \in\left[0, \frac{1}{\delta_{N}^{\theta}}\right]$. In other words, $1 / \delta_{N}^{\theta}$ is lower bound for the domain of analyticity of $\Pi_{\theta}$. 


\subsection{Numerical Example}

We apply our theoretical results to the coupled processors model. Consider a system with two finite queues, each with their own server. Customers for queue $i$ enter the system at a Poisson rate $\lambda_{i}$. As long as there are customers in both queues, the server of queue $i$ works at service rate $\mu_{i}$. However if the server of queue $i$ is idle, it joins the non-idle server $j$ making the average service rate of the non-idle queue larger than $\mu_{j}$.

There are but few analytical results for this model, see $[5,10]$ for a derivation of the moment generating function of the joint distribution. In particular, no closed form solution for stationary distribution is known. In [9] a heavytraffic approximation was found, and in [8] a large deviation analysis is given.

We take for $P$ the kernel of the system where customers arrive with rate $\lambda_{i}$ to queue $i$, and where each queue has its own server that serves at rate $\mu_{i}$, independent of the state of the other queue. We denote Markov kernel of the embedded jump chain of the coupled processor model by $Q$. The kernel $P_{\theta}$ (see (19) for the definition) represents then a system where the server of an idle queue joins the non-idle queue with probability $\theta$.

Consider the following numerical example. Take $\lambda_{1}=1.9, \lambda_{2}=1.8, \mu_{1}=$ $\mu_{2}=2, b_{1}=5$ and $b_{2}=5$. We are interested in the probability that queue 1 is full, and we call this the overflow probability. We denote the overflow probability given by $P_{\theta}$ by $\hat{p}_{\theta}$. We develop the series at $\theta=0$, that is, $D_{P_{\theta}}=D_{P}$ and $\Pi_{\theta+\Delta}=\Pi_{\Delta}$ with $\Pi_{\theta}=\Pi_{P}$. The series in (21) thus reads

$$
H_{0, \Delta}(k)=\Pi_{P} \sum_{n=0}^{k} \Delta^{n}\left((Q-P) D_{P}\right)^{n},
$$

for $k \geq 0$.

Let $\hat{p}_{\Delta}(k)$ denote the prediction of the overflow probability based on $H_{0, \Delta}(k)$. In all numerical examples $N$ turned out to be 1 . Figure 4 shows the absolute relative error (in formula, $\left.\left|\hat{p}_{\Delta}-\hat{p}_{\Delta}(k)\right| / \hat{p}_{\Delta}\right)$ of predicting the overflow probability at queue 1 by $H_{0, \Delta}(k)$ for different values of $k$ and different values for $\theta$.

Figure 5 shows the absolute relative error of the remainder term (in formula, $\left(\hat{r}_{\Delta}(k)-\left(\hat{p}_{\Delta}-\hat{p}_{\Delta}(k)\right)\right) / \hat{p}_{\Delta}$, where $\hat{r}_{\Delta}(k)$ denotes our upper bound for $\left.R_{0, \Delta}(k)\right)$ for different values of $k$ and different values of $\Delta$. In all cases, condition $\mathbf{C}(0)$ is satisfied for $N=2$ and $c_{\delta_{N}^{0}}=0.84$.

Remark 4.3 For this example it turns out that $\frac{c g_{1}}{1-\beta}=0.3342 \times 14.1940>1$. 


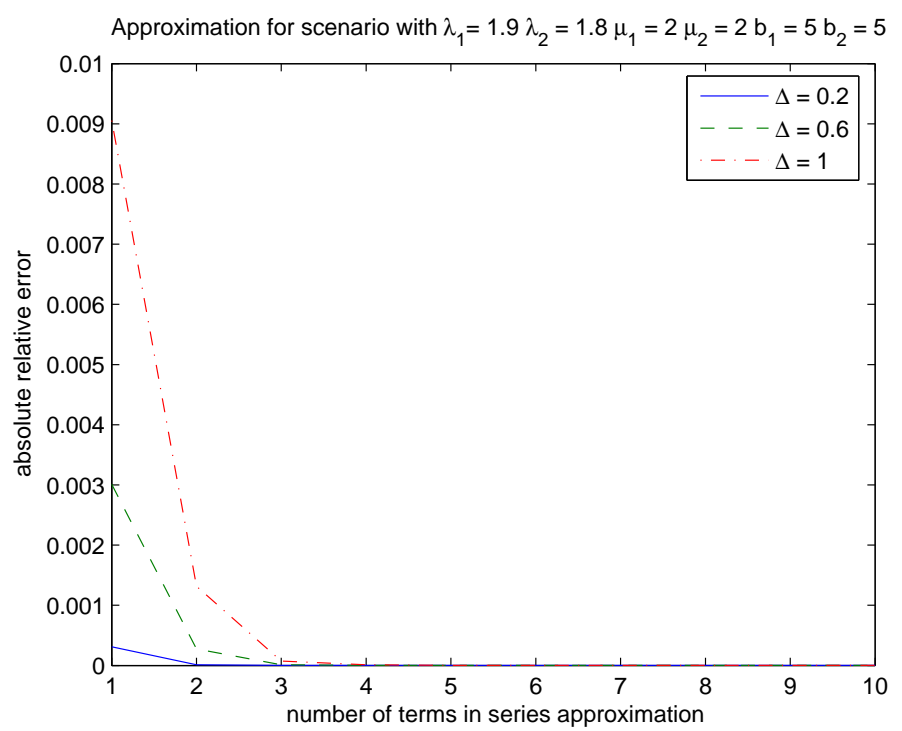

Figure 4: Absolute relative error

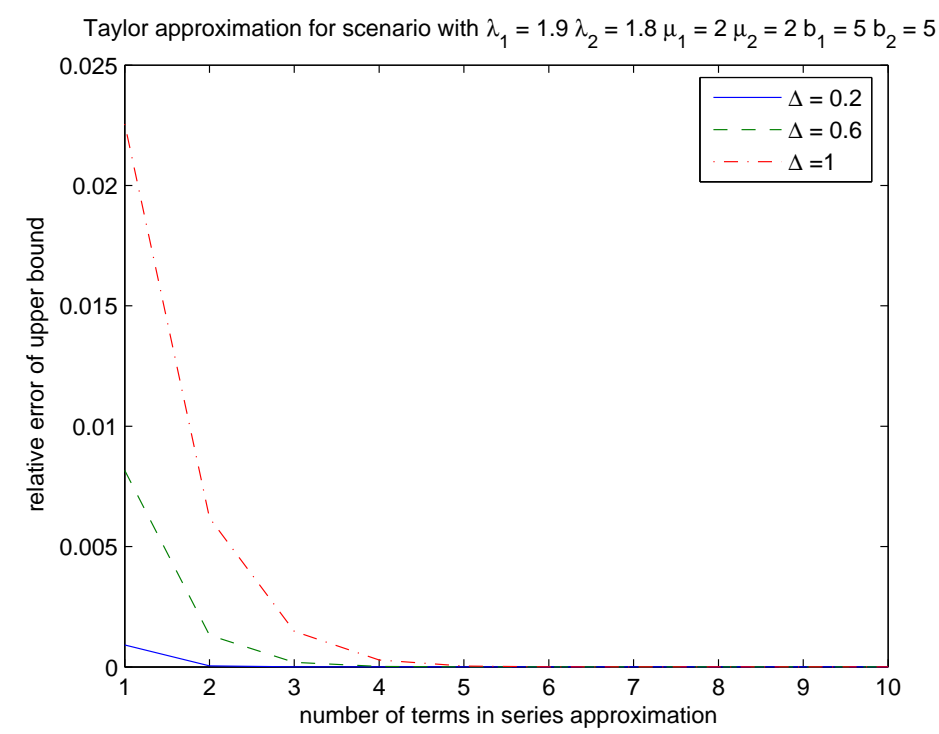

Figure 5: Relative error of the upper bound of the remainder term 


\section{Conclusion}

We presented a new algorithm for the approximative computation of characteristics of finite Markov chains. It could be shown that under quite mild conditions the algorithm approximates the true solution. Moreover, upper bounds for the error of the approximations could be established. Numerical examples illustrated that the fast convergence rate of our algorithm. Future work we will study our methodology for large scaled problems.

\section{References}

[1] E. Altman, K.E. Avrachenkov, and Núñez-Queija. Perturbation analysis for denumerable markov chains with application to queueing models. Advances in Applied Probability, 36:839-853, 2004.

[2] X.-R. Cao. The Maclaurin Series for Performance Functions of Markov Chains. Advances in Applied Probability, 30:676-692, 1998.

[3] G.E. Cho and C.D. Meyer. Comparison of perturbation bounds for the stationary distribution of a Markov chain. Linear Algebra and its Applications, 335:137-150, 2001.

[4] P. Coolen-Schrijner and E.A. van Doorn. The deviation matrix of a continuous-time markov chain. Probability in the Engineering and Informational Sciences, 16:351-366, 2002.

[5] G. Fayolle and R. Iasnogorodski. Two coupled processors: the reduction to a Riemann-Hilbert problem. Zeitschrift für Wahrscheinlichkeitstheorie und verwandte Gebiete, 47:325-351, 1979.

[6] H. Hartmann and C. Arguelles. Transience boundes for long walks. Mathematics of Operations Research, 24:414-439, 1999.

[7] B. Heidergott and A. Hordijk. Taylor series expansions for stationary Markov chains. Advances in Applied Probability, 35:1046-1070, 2003.

[8] A. Hordijk and N. Popov. Large deviation analysis of a coupled processor system. Probability in the Engineering and Informational Sciences, 17:397-409, 2003.

[9] C. Knessl and J.A. Morrison. Heavy traffic analysis of two coupled processors. Queueing Systems, 43:173-220, 2003. 
[10] A.G. Konheim, I. Meilijson, and A. Melkman. Processor sharing of two parallel lines. Journal of Applied Probability, 18:952-956, 1981.

[11] H. Minc. Nonnegative Matrices. Wiley, New York, 1988.

[12] M.L. Puterman. Markov Decision Processes: Discrete Stochastic Dynamic Programming. John Wiley \& Sons, New York, 1994.

\section{Appendix}

We give a proof of Theorem 3.7. Let $A=\left|\Pi_{P}\left((Q-P) D_{P}\right)^{k}\right|$, for $k \in \mathbb{N}$. The important case is when $A$ has non-zero elements. Indeed, if $A$ is identical to the zero matrix, then its trivial that

$$
0=\inf _{v}\|A\|_{v}=\|A\|_{1} .
$$

Now suppose that $A$ has non-zero elements. The key observation for the proof is that any two rows of $A$ are identical. Suppose that $A$ has $m$ non-zero columns. Hence, after possible re-labeling of states, $A$ is of the form

$$
A=\left(\begin{array}{cc}
A_{1} & 0 \\
A_{2} & 0_{2}
\end{array}\right)
$$

where $A_{1}$ is a $m \times m$ dimensional matrix with all elements larger than zero, $A_{2}$ is a $(S-m) \times m$ matrix with all elements larger than zero, and $0_{1}$ and $0_{2}$ are zero matrices of appropriate size.

The proof has two main steps. First, we will show that

$$
\lambda=\inf _{v}\left\|A_{1}\right\|_{v}
$$

where $\lambda$ denotes the maximal eigenvalue of $A_{1}$. In a second step, we will show that (22) holds when $A_{1}$ is replaced by the original matrix $A$.

We now turn to the proof of (22). Observe that solving (22) can be interpreted as finding the minimal value of $\left\|A_{1}\right\|_{v}$. This problem can be translated into an equivalent linear programming problem as follows. We have to find the minimal value for $\delta$ such that the following problem

$$
\begin{gathered}
\qquad \min \sum_{i \in S} v(i) \\
\text { such that }\left(A_{1}-\delta I\right) v \leq 0 \\
\text { and } v \geq 1,
\end{gathered}
$$

returns a feasible solution $v$. The following lemma shows that the minimal $\delta$ for which (23) has a solution is equal to the maximal eigenvalue of $A_{1}$. 
Lemma 5.1 The linear programming problem (23) has for any $\delta \in[\lambda, \infty)$ has a solution, where $\lambda>0$ is the maximal eigenvalue of $A_{1}$.

Proof Note that $A_{1}$ is a full matrix and thus irreducible. Furthermore, all elements of $A_{1}$ are larger than or equal to zero. Thus Theorem 4.5 in [11] (Chapter 1) applies, which gives immediately that for any $\delta$ larger than or equal to the maximal eigenvalue $\lambda$ the linear programming problem (23) has a solution. With the Perron Frobenius theorem it follows that $\lambda>0$. Finally use Corollary 4.2 and Theorem 4.4 (both are again in [11], Chapter 1) to conclude that for any $\delta$ the feasible vector $v$ is the rescaled unique positive eigenvector associated with $\lambda$.

Matrix $A_{1}$ has identical rows, thus any eigenvector associated with the maximal eigenvalue of $A$ has identical components. This implies $A_{1} \mathbf{1}=\lambda \mathbf{1}$ and, by Lemma 5.1,

$$
\lambda=\left\|A_{1}\right\|_{1}=\inf _{v}\left\|A_{1}\right\|_{v}
$$

which establishes (22).

For the second part of the proof, suppose that $(\delta, v)$ is a solution of the (overall) LP:

$$
\begin{gathered}
\min \sum_{i \in S} v(i) \\
\text { such that }(A-\delta I) v \leq 0 \\
\text { and } \quad v \geq 1 .
\end{gathered}
$$

Denote the restriction of $v$ to the first $m$ elements of $v$ by $v^{1}$ and the restriction of $v$ to the elements $m+1$ up to $S$ by $v^{2}$. Then,

$$
A_{1} v^{1} \leq \delta v^{1} \quad \text { and } \quad A_{2} v^{2} \leq \delta v^{2} .
$$

Since $\delta \geq \lambda$ and $A_{1}$ and $A_{2}$ have identical rows, we obtain

$$
\lambda=\|A\|_{1}=\inf _{v}\|A\|_{v},
$$

which proves the claim. 